\title{
UNIVERSAL ENVELOPING ALGEBRAS WITH SUBEXPONENTIAL BUT NOT POLYNOMIALLY BOUNDED GROWTH
}

\author{
MARTHA K. SMITH ${ }^{1}$
}

\begin{abstract}
ABSTRACr. The universal enveloping algebras of certain Lie algebras provide examples of integral domains with growth which is subexponential but not bounded by any polynomial.
\end{abstract}

1. Suppose the (not necessarily associative) algebra $S$ is generated over the field $k$ by the finite set $X$. Let $S(X, n)$ denote the $k$-subspace of $S$ spanned by all monomial words of length less than or equal to $n$ in the elements of $X$. The growth function $\gamma_{S}(n)$ of $S$ with respect to $X$ is defined as $\operatorname{dim} S(X, n)$.

G. Bergman has pointed out that $\lim _{n \rightarrow \infty} \gamma_{S}(n)^{1 / n}$ always exists. For clearly, $\gamma_{S}(n+m) \leqslant \gamma_{S}(n) \gamma_{S}(m)$. Thus if $n=m k+l$, where $k \geqslant 0$ and $0 \leqslant l<m$,

$$
\gamma_{S}(n)^{1 / n} \leqslant \gamma_{S}(m)^{k / n} \gamma_{S}(l)^{1 / n} \leqslant \gamma_{S}(m)^{1 / m} \gamma_{S}(m)^{1 / n}
$$

(since $\gamma_{S}$ is nondecreasing). Taking $\gamma_{S}(m)^{1 / m}$ close to lim inf $\gamma_{S}(n)^{1 / n}$ shows that $\gamma_{S}(n)^{1 / n}$ converges.

Definitions. (i) $S$ has exponential growth if $\lim \gamma_{S}(n)^{1 / n}>1$. Otherwise, $S$ has subexponential growth. (ii) $S$ has polynomially bounded growth if there exists a polynomial $p$ with $\gamma_{S}(n) \leqslant p(n)$ for all sufficiently large $n$.

If $X$ is another set of generators with corresponding growth function $\gamma_{S}^{\prime}(n)$, then $\gamma_{S}^{\prime}(n) \leqslant \gamma_{S}(m n)$ where $X^{\prime} \subseteq S(X, m)$, so that (i)-(ii) are independent of $X$.

In this note we examine the growth of universal enveloping algebras in order to show the existence of integral domains with subexponential but not polynomially bounded growth. It is not known if groups with such growth functions exist (cf. [1], [5], [6]).

Let $\lambda_{S}(n)=\gamma_{S}(n)-\gamma_{S}(n-1)$. For $s \in S$, the $X$-length of $s$ is $l_{S}(s)=$ $\min \{n \mid s \in S(X, n)\}$.

2. $S$ has subexponential growth if and only if $\lim _{\sup _{n \rightarrow \infty}} \lambda_{S}(n)^{1 / n} \leqslant 1$. Necessity is clear. To establish sufficiency, note that $\lim _{\sup _{n \rightarrow \infty}} \lambda_{S}(n)^{1 / n} \leqslant 1$ implies that the generating function $F(t)=\Sigma \lambda_{S}(n) t^{n}$ converges absolutely in the unit circle. But then so does

Received by the editors December 5, 1975.

AMS (MOS) subject classifications (1970). Primary 16A48; Secondary 16A02, 17B35, 17 B65.

Key words and phrases. Subexponential growth, polynomially bounded growth, universal enveloping algebra.

${ }^{1}$ Research supported in part by an NSF grant. 


$$
G(t)=\sum t^{m} \sum \lambda_{S}(n) t^{n}=\sum \gamma_{S}(n) t^{n},
$$

yielding the desired inequality.

3. Let $L$ denote a Lie algebra generated over the field $k$ by the finite set $X$ whose elements are linearly independent over $k$. Let $U=U(L)$ denote its universal enveloping algebra. We may assume $L \subseteq U(L)$, so that $X$ generates $U$ as an associative algebra. We may choose a basis $u_{1}, u_{2}, \ldots$ for $L$ such that $X=\left\{u_{1}, \ldots, u_{\gamma_{L}(1)}\right\}$ and such that $u_{\gamma_{L}(n-1)+1}, \ldots, u_{\gamma_{L}(n)}$ is a basis for $L(X, n)$ modulo $L(X, n-1)$. By the Birkhoff-Poincaré-Witt Theorem [4, p. 159], the "standard" monomials $u_{i_{1}} u_{i_{2}} \cdots u_{i_{r}}$ with $i_{1} \leqslant i_{2} \leqslant \cdots \leqslant i_{r}$ form a basis for $U$.

4. Clearly $l_{U}\left(u_{i}\right) \leqslant l_{L}\left(u_{i}\right)$. Define the "formal length" $\bar{l}$ of an arbitrary monomial $u_{i_{1}} \cdots u_{i_{r}}$ to be $\sum_{j=1}^{r} l_{L}\left(u_{i_{1}}\right)$. It is easily seen that the canonical process [4, p. 157] for rewriting a monomial as a linear combination of standard monomials produces monomials of standard length no greater than that of the original. It follows that $l_{U}\left(u_{i}\right)=l_{L}\left(u_{i}\right)$ (henceforth denoted $l\left(u_{i}\right)$ ), and more generally that the length in $U$ of a standard monomial is its formal length. Consequently, $\lambda_{U}(n)$ is the cardinality of the set

$$
\left\{\left(\mu_{1}, \ldots, \mu_{\gamma_{L}(n)}\right) \mid \sum \mu_{i} l\left(u_{i}\right)=n, \mu_{i} \text { nonnegative integers }\right\},
$$

so that

$$
F(t)=\prod_{i=1}^{\infty}\left(1-t^{l\left(u_{i}\right)}\right)^{-1}=\sum_{n=0}^{\infty} \lambda_{U}(n) t^{n}
$$

is a generating function for $\lambda_{U}(n)$.

5. If $L$ is finite dimensional, we may choose $X$ to be a basis for $L$. Then by 4, $\gamma_{U}(n)$ is the number of $r$-tuples $\left(\mu_{1}, \ldots, \mu_{r}\right)$ of nonnegative integers ( $r=\operatorname{dim} L$ ) with $\sum \mu_{i} \leqslant n$. The argument of [6, Proposition 3.6] then shows

$$
\gamma_{U}(n)=\sum_{i=0}^{r}\left(\begin{array}{l}
r \\
i
\end{array}\right)\left(\begin{array}{c}
n \\
i
\end{array}\right)
$$

which for $n \geqslant r$ is a polynomial of degree $r$ in $n$ (cf. [2], where growth is studied by considering $\lim \sup [(\log \gamma(n)) / \log n]$.

6. If $L$ is not finite dimensional, $U$ does not have polynomially bounded growth. For in the notation of 3 , there exists for each $n>0$ some $u_{i}$ with $l\left(u_{i_{j}}\right)=n$. The number of standard monomials in the $u_{i,}$ 's with length $n$ is then $p(n)$, the number of partitions of $n$. Since [3, p. 237] for any $\varepsilon>0$, $\exp ((\pi \sqrt{2} / 3-\varepsilon) \vee n)<p(n), \gamma_{U}(n)$ cannot be bounded above by any polynomial.

7. If $L$ has subexponential growth, then so does $U$. For as in 2 , it suffices to show that $F(t)=\sum_{n=0}^{\infty} \lambda_{U}(n) t^{n}$ has radius of convergence at least 1 . By 4 ,

$$
F(t)=\prod_{i=1}^{\infty}\left(1+\frac{t^{l\left(u_{i}\right)}}{1-t^{l\left(u_{i}\right)}}\right) .
$$


Then $F$ converges absolutely in the unit circle if and only if the series $\sum_{i=1}^{\infty} r^{l\left(u_{l}\right)} /\left(1-r^{l\left(u_{i}\right)}\right)$ converges for all $0<r<1$. Ratio comparison with the convergent series $\sum_{i=1}^{\infty} r^{l\left(u_{i}\right)}=\sum_{n=1}^{\infty} \lambda_{L}(n) r^{n}$ shows that it does.

8. There exist finitely-generated infinite-dimensional Lie algebras with subexponential growth, e.g., the Lie algebra $L$ with basis $x, y_{1}, y_{2}, \ldots$ such that $\left[x, y_{i}\right]=y_{i+1},\left[y_{i}, y_{j}\right]=0$. Thus there exist finitely-generated associative algebras with growth which is neither polynomially bounded nor exponential. The examples obtained in the above manner are domains $[4$, p. 166].

\section{REFERENCES}

1. H. Bass, The degree of polynomial growth of finitely generated nilpotent groups, Proc. London Math. Soc. 25 (1972), 603-614.

2. I. M. Gel'fand and A. A. Kirillov, Sur les corps liés aux algèbres enveloppantes des algèbres de Lie, Inst. Hautes Études Sci. Publ. Math. No. 31 (1966), 5-19. MR 34 \#7731.

3. E. Grosswald, Topics from the theory of numbers, Macmillan, New York, 1966. MR 37 \#3989.

4. N. Jacobson, Lie algebras, Interscience Tracts in Pure and Appl. Math., no. 10, Interscience, New York, 1962. MR 26 \# 1345.

5. J. Milnor, Problem 5603, Amer. Math. Monthly 75 (1968), 685-686.

6. J. A. Wolf, Growth of finitely generated solvable groups and curvature of Riemannian manifolds, J. Differential Geometry 2 (1968), 421-446. MR 40 \# 1939.

Department of Mathematics, University of TeXas, Austin, TeXas 78705 